\title{
Numerical Solution for a Similar Flow between Two Disks in the Presence of a Magnetic Field
}

\author{
Sajjad Hussain ${ }^{1}$, Muhammad Anwar Kamal', Farooq Ahmad ${ }^{1}$, Muhammad Ali ${ }^{3}$, \\ Muhammad Shafique ${ }^{4}$, Sifat Hussain ${ }^{1}$ \\ ${ }^{1}$ Centre for Advanced Studies in Pure and Applied Mathematics, Bahauddin Zakariya University, Multan, Pakistan \\ ${ }^{2}$ Department of Mathematics, King Suleman Bin Abdulaziz University, Al-Kharj, Saudi Arabia \\ ${ }^{3}$ Department of Mathematics, Minhaj University, Lahore, Pakistan \\ ${ }^{4}$ Department of Mathematics, Gomal University, D. I. Khan, Pakistan \\ Email: sajjad_h96@yahoo.com,makamal@yahoo.com,farooqgujar@gmail.com,Muhammadalimaths@gmail.com, \\ mshafique6161@hotmail.com, siffat2002@gmail.com
}

Received May 19, 2013; revised June 19, 2013; accepted June 26, 2013

Copyright (C) 2013 Sajjad Hussain et al. This is an open access article distributed under the Creative Commons Attribution License, which permits unrestricted use, distribution, and reproduction in any medium, provided the original work is properly cited.

\begin{abstract}
Numerical solutions are obtained for non-steady, incompressible fluid flow between two parallel disks which at time $t$ are separated by a distance $H(1-\alpha t)^{1 / 2}$ and a magnetic field proportional to $B_{0}(1-\alpha t)^{-1 / 2}$ is applied perpendicular to the disks where $H$ denotes a representative length, $B_{0}$ denotes a representative magnetic field and $\alpha^{-1}$ denotes a representative time. Similarity transformations are used to convert the governing partial differential equations of motion into ordinary differential form. The resulting ordinary differential equations are solved numerically using SOR method, Richardson extrapolation and Simpson's (1/3) Rule. Our numerical scheme is straightforward, efficient and easy to program.
\end{abstract}

Keywords: Numerical Solution; Similar Flow; Newtonian Fluid; Magnetic Field; Reynold's Number

\section{Introduction}

The quest for similar solutions is particularly important with respect to the mathematical character of the solution. In cases where similar solutions exist, it is possible, to reduce the system of partial differential equations to one involving ordinary differential equations, which evidently constitute, a considerable mathematical simplifycation of the problem. Wang [1] studied a viscous fluid between two parallel plates, which are being squeezed or separated with normal velocity proportional to $(1-\alpha t)^{-1 / 2}$ and found similarity solutions of the unsteady Navier-Stocks equations. Ishizawa [2] derived a similarity solution to the case of the unsteady laminar flow between two parallel disks. Tichy and Bourgin [3] found that a similarity solution does exist for the steady flow in a narrow channel of a gap width varying as $(a+b x)^{m}$, where $a, b$ and $m$ are constants. Bhupendra et al. [4] considered the problem of forced flow of an electrically conducting viscous incompressible fluid due to an infinite rotating disk under the influence of uniform magnetic field, applied normal to the flow. Pavlov [5] found an exact similarity solution of MHD boundary layer equations for the steady two dimensional flow of an electrically conducting incompressible fluid due to rotation of a plane elastic surface in the presence of a uniform transverse magnetic field. Guria et al. [6] obtained exact solution of hyderomagnetic flow between two porous disks rotating with same angular velocity about two non coincident axes in the presence of a uniform transverse magnetic field. Attia [7] studied the problem of steady flow and heat transfer of a conducting fluid due to the rotation of an infinite, non conducting porous disk in the presence of an external magnetic field. Sajid et al. [8] examined the MHD rotating flow of a viscous fluid over a shrinking sheet. Asir et al. [9] gave a new hybrid analytical algorithm to study the effects of uniform suction of a laminar, steady, incompressible magnetohyderodynamic electrically conducting fluid over a rotating disk. The purpose of present study is to obtain numerical solution for similar flows of a Newtonian fluid between two disks in the presence of a magnetic field. Usha and Vasudevan [10] studied a similar flow between two rotating disks in the presence of a magnetic field and obtained rather expensive solution of the problem to observe the effect of flow parameters on the velocity fluid. 


\section{Mathematical Analysis}

It has been assumed the flow is axisymmetric, incomepressible and non-steady. The flow is between two parallel infinite disks, which are separated a distance $h(t)$ apart, where $t$ denotes time. A magnetic field of strength $B(t)$ is applied perpendicular to the two disks. The upper disk is moving with velocity $h^{\prime}(t)$ towards the lower fixed disk. Cylindrical polar coordinates $(r, \theta, z)$ are used. The lower disk is at $z=0$ and the upper one is at $z=h(t)$ where $h(0)=H$.

The equations of motion in component form become as follows:

$$
\begin{gathered}
u_{r}+\frac{u}{r}+\frac{1}{r} v_{\varphi}+w_{z}=0 \\
u u_{r}+\frac{v}{r} u_{\varphi}+w u_{z}-\frac{v^{2}}{r}+\frac{\delta B^{2}}{\rho} u \\
=-\frac{1}{\rho} p_{r}+v\left\{u_{r r}+\frac{1}{r} u_{r}-\frac{u}{r^{2}}+\frac{1}{r^{2}} u_{\varphi \varphi}-\frac{2}{r^{2}} v_{\varphi}+u_{z z}\right\} \\
u v_{r}+\frac{v}{r} v_{\varphi}+w v_{z}+\frac{u v}{r}+\frac{\delta B^{2}}{\rho} v \\
=-\frac{1}{r \rho} p_{\varphi}+v\left(v_{r r}+\frac{1}{r} v_{r}-\frac{v}{r^{2}}+\frac{1}{r^{2}} v_{\varphi \varphi}+\frac{2}{r^{2}} u_{\varphi}+v_{z z}\right) \\
u w_{r}+\frac{v}{r} w_{\varphi}+w w_{z}=-\frac{1}{\rho} p_{z}+v\left\{w_{r r}+\frac{1}{r} w_{r}+\frac{1}{r^{2}} w_{\varphi \varphi}+w_{z z}\right\}
\end{gathered}
$$

where the subscripts denote the partial differentiation with respect to space coordinates $\rho$ is the density, $p$ the pressure and $v$ the coefficient of kinematics viscosity.

The boundary conditions are:

$$
\begin{aligned}
& u=0, w=0, \quad \text { at } z=0 \\
& u=0, w=h^{\prime}, \text { at } z=h(t)
\end{aligned}
$$

The following similarity transformations are used:

$$
\begin{gathered}
u=\frac{\alpha r}{2(1-\alpha t)} f^{\prime}(y) \\
w=\frac{-\alpha H}{\sqrt{1-\alpha t}} f(y) \\
B=\frac{B_{0}}{\sqrt{1-\alpha t}}
\end{gathered}
$$

where

$$
y=\frac{z}{H \sqrt{1-\alpha t}}
$$

is the dimensionless variable. The equation of continuity is identically satisfied. Equations (2) and (3) take the forms below respectively.

$$
\begin{aligned}
& \frac{1}{r} \frac{\partial p}{\partial r} \\
& =\frac{\rho \alpha^{2}}{4(1-\alpha t)^{2}}\left(\frac{1}{\operatorname{Re}} f^{\prime \prime \prime}-y f^{\prime \prime}-2 f^{\prime}-\left(f^{\prime}\right)^{2}+2 f f^{\prime \prime}-M^{2} f^{\prime}\right) \\
& \frac{\partial p}{\partial y}=\frac{-\rho \alpha^{2} H^{2}}{2(1-\alpha t)}\left(\frac{1}{\operatorname{Re}} f^{\prime \prime}-y f^{\prime}-f+2 f f^{\prime}\right)
\end{aligned}
$$

where $\operatorname{Re}=\frac{\rho \alpha H^{2}}{2 \mu}, M^{2}=\frac{B_{0}^{2} \sigma}{\mu}$, here $\mu$ denotes viscosity and $\sigma$ denotes fluid electrical conductivity. Whence the differentiation of Equation (6) with respect $y$ and that of Equation (7) with respect to $r$ yield:

$$
\operatorname{Re}\left(y f^{\prime \prime \prime}+3 f^{\prime \prime}-2 f f^{\prime \prime \prime}\right)+M^{2} f^{\prime \prime}=f^{i v}
$$

Equation (8) is integrated to get:

$$
\operatorname{Re}\left(y f^{\prime \prime}+2 f^{\prime}-2 f f^{\prime \prime}+\left(f^{\prime}\right)^{2}\right)+M^{2} f^{\prime}=f^{\prime \prime \prime}+\beta
$$

where $\beta$ is constant of integration.

The boundary conditions in dimensionless form become;

$$
\begin{array}{ll}
f=0, f^{\prime}=0 & \text { at } y=0 \\
f=0.5, f^{\prime}=0 & \text { at } y=1
\end{array}
$$

\section{Finite-Difference Equations}

In order to solve Equation (9) numerically, we let

$$
q=f^{\prime}
$$

and we obtain

$$
\operatorname{Re}\left(f q^{\prime}+2 q-2 f q^{\prime}+q^{2}\right)+M^{2} q=q^{\prime \prime}+\beta
$$

The boundary conditions (10) become as:

$$
\begin{array}{ll}
f=0, q=0 & \text { at } y=0 \\
f=0.5, q=0 & \text { at } y=1
\end{array}
$$

The derivatives involved in Equation (12) are approximated by central difference approximation at a typical point $y=y_{n}$ of interval $[0,1]$, we get.

$$
\begin{aligned}
& \left(1-\operatorname{Re} h\left(\frac{y}{2}-f_{n}\right)\right) q_{n+1}-\left(2+h^{2} M^{2}+h^{2} R\left(2+q_{n}\right)\right) q_{n} \\
& +\left(1+\operatorname{Re} h\left(\frac{y}{2}-f_{n}\right)\right) q_{n-1}+h^{2} \beta=0
\end{aligned}
$$

where $h$ denotes a grid size and Equation (11) is integrated numerically. Also the symbols used denote $q_{n}=q\left(y_{n}\right)$ and $f_{n}=f\left(y_{n}\right)$.

\section{Computational Procedure}

Finite difference Equation (14) and the first order ordi- 
nary differential Equation (11) are solved simultaneously by using SOR method Smith [11, p.262] and Simpson's $(1 / 3)$ rule Gerald [12, p.293] with the formula given in Milne [13, p.48] respectively subject to the appropriate boundary conditions.

The order of the sequence of iterations is as follows:

1) The Equation (14) for the solution of $q$ is solved subject to the boundary conditions (13).

2) For the solution of $f$ we use the computed values of $q=f^{\prime}$ from above step in to Equation (11) and integrate by Simpson's (1/3) rule.

3) The optimum value of the relaxation parameter $\omega_{\text {opt }}$ is estimated to accelerate the convergence of the SOR method.

4) The SOR procedure is terminated when the following criterion is satisfied for $q$ :

$$
\max _{i=1}^{n}\left|U_{i}^{n+1}-U_{i}^{n}\right|<10^{-6},
$$

where $n$ denotes the number of iterations and $U$ stands for $q$.

The above steps 1 to 4 are repeated for higher grid levels $h / 2$ and $h / 4$. The SOR procedure gives the solution of $q=f^{\prime}$ of order of accuracy $O\left(h^{2}\right)$ due to second order finite differences used to approximate the derivatives while Simpson's (1/3) rule gives the order of accuracy $O\left(h^{5}\right)$ in the solution of $f$. Higher order accuracy in the solution of $q=f^{\prime}$ on the basis of above solutions is achieved by using Richardson's Extrapolation Burden [14, p.168]. The solution of order of accuracy $O\left(h^{6}\right)$ in the following tables for computation of $q=f^{\prime}$ is the most accurate and accepted solution.

\section{Results and Discussion}

The numerical solutions examine the way in which the flow pattern changes with the squeeze Reynolds number Re and Hartmann number M. Hamza [15] investigated this problem for the range $0.0<\operatorname{Re} \leq 10.0,0.0<M \leq$ 30.0. We extended the previous work and analyzed the problem for the parameters involved in the range $0.0<\operatorname{Re} \leq 20.0$ and $0.0<M \leq 50.0$.

Table 1 shows the values of different parameters used in the numerical procedure. The numerical results for $f, f^{\prime}$ have been computed for different values of flow parameters namely Re and $M$. The accuracy of numerical results is checked by comparing the results on three different grid sizes namely $h=0.025,0.012$ and 0.006 . The comparison of $f^{\prime}$ is shown in the Tables 24 using Richardson extrapolation method. Graphically, the results have been demonstrated in Figures 1-6. It is found that for fixed Re, there is a slight increase in the

Table 1. Optimum value of relaxation parameter used in SOR method.

\begin{tabular}{|c|c|c|c|c|c|c|c|c|}
\hline \multirow{3}{*}{$\operatorname{Re}$} & \multirow{3}{*}{$M$} & \multirow{3}{*}{$\beta$} & \multicolumn{6}{|c|}{ Number of Iterations in SOR method with $\omega_{\text {opt }}$} \\
\hline & & & \multicolumn{2}{|c|}{$h=0.025$} & \multicolumn{2}{|c|}{$h=0.012$} & \multicolumn{2}{|c|}{$h=0.006$} \\
\hline & & & $\mathrm{NI}$ & $\omega_{\text {opt }}$ & $\mathrm{NI}$ & $\omega_{\text {opt }}$ & NI & $\omega_{o p t}$ \\
\hline \multirow{3}{*}{0.01} & 10 & 62.5154 & 38 & 1.60 & 122 & 1.70 & 282 & 1.80 \\
\hline & 30 & 482.206 & 18 & 1.80 & 40 & 1.85 & 96 & 1.90 \\
\hline & 50 & 1302.32 & 17 & 1.70 & 65 & 1.75 & 88 & 1.85 \\
\hline \multirow{3}{*}{5.0} & 0 & 14.10885 & 75 & 1.70 & 322 & 1.75 & 678 & 1.85 \\
\hline & 4 & 23.24505 & 72 & 1.70 & 216 & 1.75 & 469 & 1.85 \\
\hline & 8 & 49.98825 & 43 & 1.70 & 108 & 1.75 & 121 & 1.90 \\
\hline \multirow{2}{*}{15.0} & 5 & 43.1535 & 49 & 1.40 & 99 & 1.60 & 185 & 1.80 \\
\hline & 10 & 83.81528 & 54 & 1.40 & 58 & 1.60 & 243 & 1.95 \\
\hline \multirow{2}{*}{20} & 0 & 36.7535 & 44 & 1.70 & 102 & 1.75 & 126 & 1.90 \\
\hline & 10 & 90.8242 & 53 & 1.70 & 79 & 1.80 & 155 & 1.90 \\
\hline
\end{tabular}

Table 2. $M=40.0, \operatorname{Re}=0.01, M=50.0, \operatorname{Re}=0.01$.

\begin{tabular}{|c|c|c|c|c|c|c|c|c|}
\hline$y$ & $h=0.025 \quad f^{\prime}$ & $h=0.012 \quad f^{\prime}$ & $h=0.006 \quad f^{\prime}$ & Extrapolated $f^{\prime}$ & $h=0.025 \quad f^{\prime}$ & $h=0.012 \quad f^{\prime}$ & $h=0.006 \quad f^{\prime}$ & Extrapolated $f^{\prime}$ \\
\hline 0.0 & 0.000000 & 0.000000 & 0.000000 & 0.000000 & 0.000000 & 0.000000 & 0.000000 & 0.000000 \\
\hline 0.2 & 0.526146 & 0.526196 & 0.526205 & 0.526208 & 0.526146 & 0.526196 & 0.526205 & 0.526208 \\
\hline 0.4 & 0.526388 & 0.526388 & 0.526389 & 0.526389 & 0.526388 & 0.526388 & 0.526389 & 0.526389 \\
\hline 0.6 & 0.528224 & 0.526388 & 0.526388 & 0.526429 & 0.528224 & 0.526388 & 0.526388 & 0.526429 \\
\hline 0.8 & 0.526091 & 0.526197 & 0.526208 & 0.526211 & .526091 & 0.526197 & 0.526208 & 0.526211 \\
\hline 1.0 & 0.000000 & 0.000000 & 0.000000 & 0.000000 & 0.000000 & 0.000000 & 0.000000 & 0.000000 \\
\hline
\end{tabular}


Table 3. $M=10.0, \operatorname{Re}=10.0, M=0.0, \operatorname{Re}=15.0$.

\begin{tabular}{|c|c|c|c|c|c|c|c|c|}
\hline$y$ & $h=0.025 \quad f^{\prime}$ & $h=0.012 \quad f^{\prime}$ & $h=0.006 \quad f^{\prime}$ & Extrapolated $f^{\prime}$ & $h=0.025 \quad f^{\prime}$ & $h=0.012 \quad f^{\prime}$ & $h=0.006 \quad f^{\prime}$ & Extrapolated $f^{\prime}$ \\
\hline 0.0 & 0.000000 & 0.000000 & 0.000000 & 0.000000 & 0.000000 & 0.000000 & 0.000000 & 0.000000 \\
\hline 0.2 & 0.541581 & 0.542817 & 0.543309 & 0.543490 & 0.510636 & 0.514646 & 0.516699 & 0.517477 \\
\hline 0.4 & 0.600984 & 0.601243 & 0.601333 & 0.601366 & 0.654030 & 0.655700 & 0.656898 & 0.657367 \\
\hline 0.6 & 0.601583 & 0.601533 & 0.601479 & 0.601457 & 0.659464 & 0.658411 & 0.658082 & 0.657966 \\
\hline 0.8 & 0.544910 & 0.544446 & 0.544130 & 0.544007 & 0.525608 & 0.522125 & 0.520289 & 0.519591 \\
\hline 1.0 & 0.000000 & 0.000000 & 0.000000 & 0.000000 & 0.000000 & .000000 & 0.000000 & 0.000000 \\
\hline
\end{tabular}

Table 4. $M=10.0, \operatorname{Re}=15.0, M=5.0, \operatorname{Re}=20.0$.

\begin{tabular}{|c|c|c|c|c|c|c|c|c|}
\hline$y$ & $h=0.025 \quad f^{\prime}$ & $h=0.012 \quad f^{\prime}$ & $h=0.006 \quad f^{\prime}$ & Extrapolated $f^{\prime}$ & $h=0.025 \quad f^{\prime}$ & $h=0.012 \quad f^{\prime}$ & $h=0.006 \quad f^{\prime}$ & Extrapolated $f^{\prime}$ \\
\hline 0.0 & 0.000000 & 0.000000 & 0.000000 & 0.000000 & 0.000000 & 0.000000 & 0.000000 & 0.000000 \\
\hline 0.2 & 0.542199 & 0.543740 & 0.544404 & 0.544650 & 0.528226 & 0.531500 & 0.533022 & 0.533591 \\
\hline 0.4 & 0.596544 & 0.596838 & 0.596932 & 0.596965 & 0.624295 & 0.625261 & 0.625685 & 0.625843 \\
\hline 0.6 & 0.597276 & 0.597190 & 0.597125 & 0.597100 & 0.627317 & 0.626752 & 0.626428 & 0.626304 \\
\hline 0.8 & 0.546665 & 0.545932 & 0.545486 & 0.545313 & 0.539719 & 0.537186 & 0.535850 & 0.535342 \\
\hline 1.0 & 0.000000 & 0.000000 & 0.000000 & 0.000000 & 0.000000 & .000000 & 0.000000 & 0.000000 \\
\hline
\end{tabular}

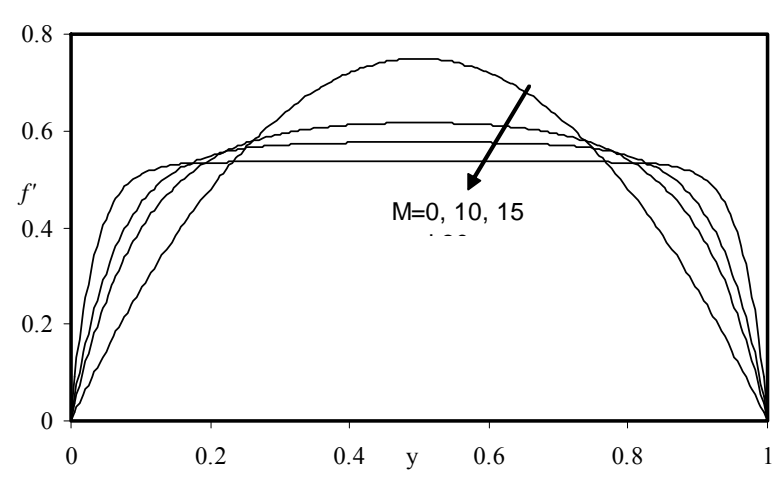

Figure 1. Graph of $f^{\prime}$ for different values of $M$ when $\mathrm{Re}=$ 0.01.

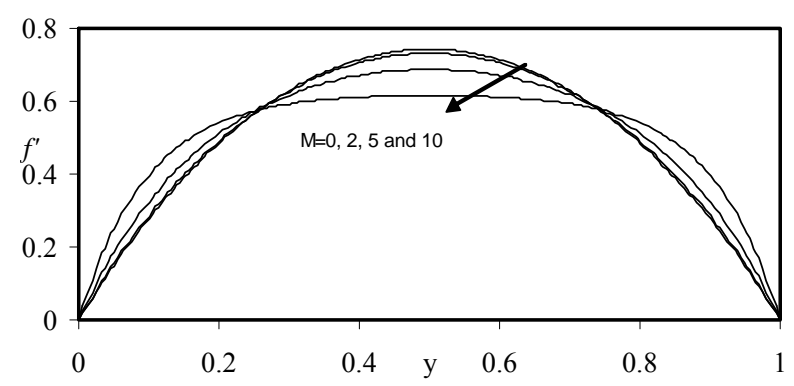

Figure 2. Graph of $f^{\prime}$ for different values of $M$ when $\mathrm{Re}=$ 1.0.

value of $f^{\prime}$ near the disks and a slight decrease in the region of the mid plane with increase in $M$. This in-

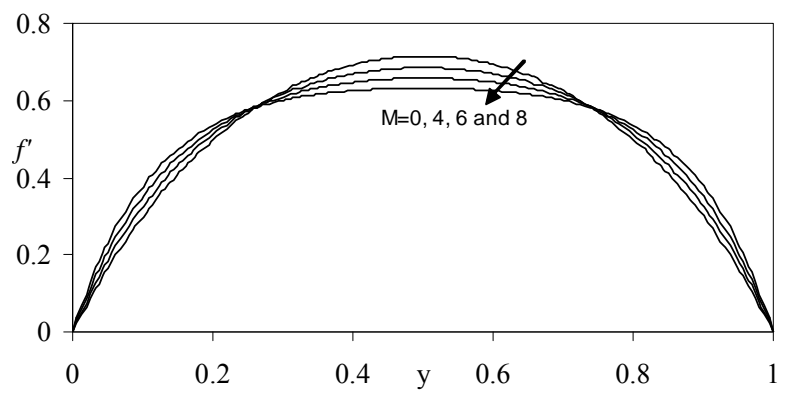

Figure 3. Graph of $f^{\prime}$ for different values of $M$ when $\operatorname{Re}=$ 5.0.

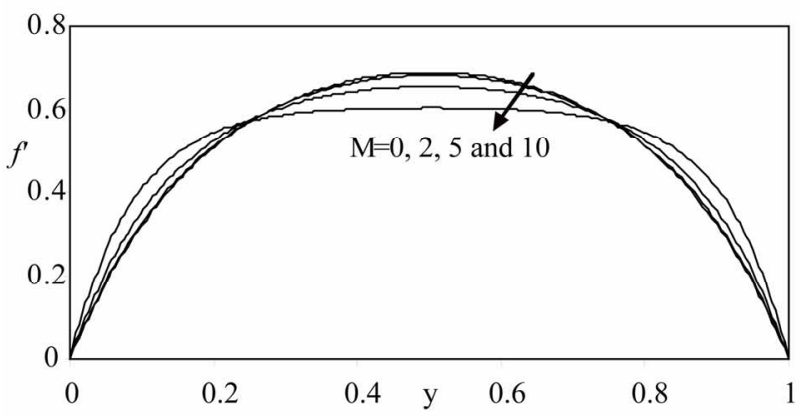

Figure 4. Graph of $f^{\prime}$ for different values of $M$ when $\operatorname{Re}=$ 10.0.

crease and decrease become more prominent with more increase in $M$, also the radial velocity profiles become more flat in the interior region for all values of Re. On 


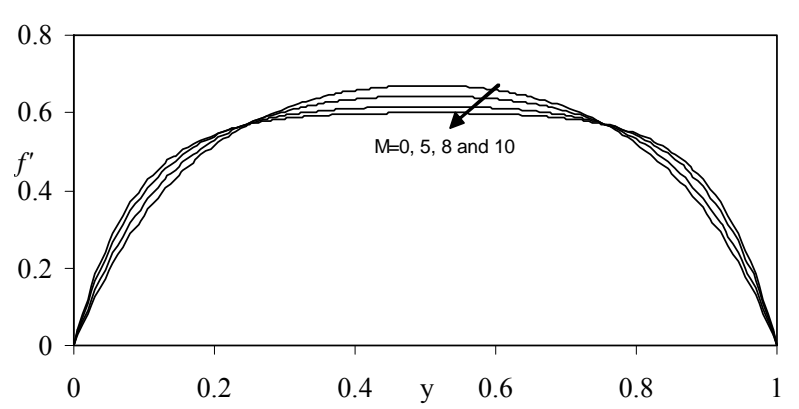

Figure 5. Graph of $f^{\prime}$ for different values of $M$ when $\mathrm{Re}=$ 15.0.

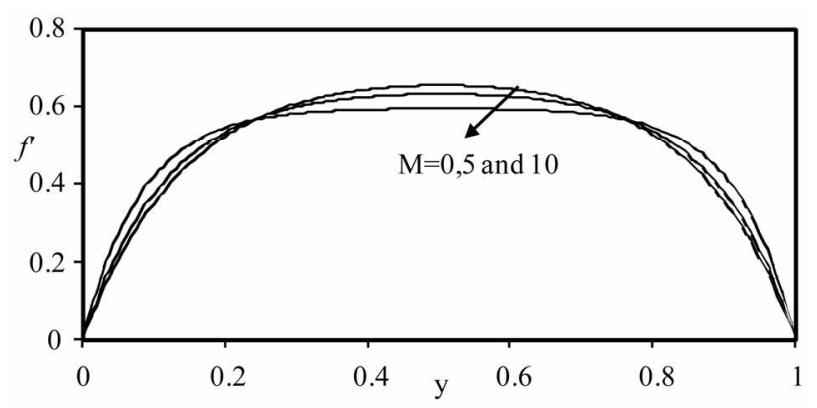

Figure 6. Graph of $f^{\prime}$ for different values of $M$ when $\mathrm{Re}=$ 20.0.

the other hand, for fixed values of $M$, the magnitude of radial flow decreases with increase in Re. The value of $\beta$ is determined by hit and trial to satisfy the approximate zero-order perturbation results given in [15] as $f=y^{2}(3 / 2-y)$.

\section{REFERENCES}

[1] C. Y. Wang, "The Squeezing of a Fluid between Two Plates," Journal of Applied Mechanics, Vol. 43, No. 4, 1976, pp. 579-583. doi:10.1115/1.3423935

[2] S. Ishizawa, "The Unsteady Laminar Flow between Two Parallel Disks with Arbitrarily Varying Gap Width," Bulletin of JSME, Vol. 9, No. 35, 1966, pp. 533-550. doi:10.1299/jsme1958.9.533

[3] J. A. Tichy and P. Bourgin, "A Similarity Solution for Flow in a Narrow Channel of Varying Gap," ASME Journal of Applied Mechanics, Vol. 53, No. 4, 1986, pp. 943-
946. doi: $10.1115 / 1.3171885$

[4] K. S. Bhupendera, K. J. Abhay and R. C. Chaudhary, "MHD Forced Flow of a Conducting Viscous Fluid through a Porous Medium Induced by an Impervious Rotating Disk Ram," Journal of Physics, Vol. 52, No. 1, 2007, pp. 73-84.

[5] K. B. Pavlov, "Magnetohydrodynamic Flow of an Incompressible, Viscous Fluid Caused by the Deformation of a Plane Surface," Magnitnaya Gidrodinamika, Vol. 4, 1974, pp. 146-147.

[6] M. Guria, B. K. Das, R. N. Jana and C. E. Imark, "Hyderomagnetic Flow between Two Porous Disks Rotating about Non Coincident Axes," Acta Mechanica Sinica, Vol. 24, No. 5, 2008, pp. 489-496. doi:10.1007/s10409-008-0158-x

[7] H. A. Attia, "On the Effectiveness of the Ion Slip on the Steady Flow of a Conducting Fluid Due to a Porous Rotating Disk with Heat Transfer," Tamkang Journal of Science and Engineering, Vol. 9, No. 3, 2006, pp. 185213.

[8] M. Sajid, T. Javed and T. Hayat, "MHD Rotating Flow of a Viscous Fluid over a Shrinking Surface," Nonlinear Dynamics, Vol. 51, No. 1-2, 2008, pp. 259-265. doi:10.1007/s11071-007-9208-3

[9] B. El-Asir, A. Mansoor, S. Bataineh and R. Arar, "A New Hybrid Analytical Analysis of the Magnetohydrodynamic Flow over a Rotating Disk under Uniform Suction," Journal of Applied Sciences, Vol. 6, No. 5, 2006, pp. 10591065. doi:10.3923/jas.2006.1059.1065

[10] R. Usha and S. Vasudevan, "A Similar Flow between Two Rotating Disks in the Presence of a Magnetic Field," Journal of Applied Mechanics, Vol. 60, No. 3, 1993, pp. 707-714. doi: $10.1115 / 1.2900862$

[11] G. D. Smith, "Numerical Solution of Partial Differential Equation," Clarendon Press, Oxford, 1979.

[12] C. F. Gerald, "Applied Numerical Analysis," AddisonWesley Pub., New York, 1989.

[13] W. E. Milne, "Numerical Solution of Differential Equation," Dover Pub., Dover, 1970.

[14] R. L. Burden, "Numerical Analysis," Prindle, Weber \& Schmidt, Boston, 1985.

[15] E. A. Hamza, "A Similar Flow between Two Disks in the Presence of a Magnetic Field," IC/87/287, Trieste, 1987. 